\title{
Conhecimento Especializado do Professor de Matemática em Dissertações e Teses Brasileiras
}

\author{
Mathematics Teacher Specialized Knowledge in Brazilian Dissertations and Theses
}

\author{
André Lima Rodrigues ${ }^{\mathrm{a}}$; Bruno Rodrigo Teixeira ${ }^{\mathrm{b}}$ \\ anniversidade Estadual de Londrina, Programa de Pós-Graduação em Ensino de Ciências e Educação Matemática. PR, Brasil \\ ${ }^{b}$ Universidade Estadual de Londrina, Departamento de Matemática. PR. Brasil. \\ *E-mail:
}

\begin{abstract}
Resumo
O conhecimento do professor é fundamental para o ensino e para a aprendizagem dos estudantes e possui, em suas características, o fato de ser especializado. Tendo isso em vista, o presente artigo tem por objetivo investigar a utilização do modelo denominado Conhecimento especializado do Professor de Matemática (MTSK) em dissertações e teses brasileiras. Para isso, foi realizada uma busca no Catálogo de Dissertações e Teses da Coordenadoria de Aperfeiçoamento de Pessoal de Nível Superior (CAPES) e na Biblioteca Digital Brasileira de Teses e Dissertações (BDTD) e o corpus foi composto por sete trabalhos. Os resultados permitiram evidenciar a utilização analítica do modelo para identificar e caracterizar conhecimentos de professores e futuros professores relacionados aos diferentes assuntos; a utilização do modelo para a realização de um trabalho de metassíntese; e também para a proposição de um modelo de conhecimento semelhante para a disciplina de Física. Ainda, destaca-se a predominância de sua utilização em contextos de formação continuada e no ensino a distância, e a ausência de trabalhos que investigam o MTSK na modalidade presencial da formação inicial. Com isso, considera-se que o estudo pode provocar questionamentos que orientem o desenvolvimento de novas pesquisas em Educação Matemática, indicando possibilidades do uso do modelo e a necessidade de valorização da profissionalização docente e da defesa de políticas públicas que reafirmem que o conhecimento do professor é especializado.
\end{abstract}

Palavras-chave: Educação Matemática. Formação de Professores. Conhecimentos Profissionais Docentes. MTSK.

\begin{abstract}
Teacher's knowledge is fundamental for the studentss' teaching and learning and has in its characteristics the fact of being specialized. With this in view, this article aims to investigate the use of the Mathematics Teacher's Specialized Knowledge (MTSK) model in Brazilian dissertations and theses. For this purpose, a search was made in the Dissertations and Thesis Catalogue of the Coordination for the Improvement of Higher Education Personnel (CAPES) and the Brazilian Digital Library of Theses and Dissertations (BDTD) and the corpus comprised seven papers. The results highlighted the analytical use of the model to identify and characterize the teachers' and future teachers' knowledge related to different subjects; the use of the model for performing a meta-synthesis work; and also, for proposing a similar knowledge model for the Physics subject. Additionally, the predominance of its use is highlighted in contexts of continuous education and in distance education, and the absence of works that investigate MTKS in face-to-face modality of initial formation. Therefore, it is considered that the study may bring about questions that may guide the development of new research in Mathematics Education, indicating possibilities of using the model and the need to value teaching professionalization and the defense of public policies reaffirming that the teacher's knowledge is specialized.
\end{abstract}

Keywords: Mathematics Education. Teacher's education. Teacher's professional knowledge. MTSK.

\section{Introdução}

Atuando como mediador do conhecimento, em sala de aula, por meio do ensino o professor de Matemática pode oportunizar situações para que o aluno desenvolva habilidades relacionadas à representação, compreensão, investigação, comunicação, contextualização (BRASIL, 2002, 2006), e para que o aluno construa seu conhecimento matemático. Desse modo, o conhecimento do professor influencia, diretamente, nos processos de ensino e de aprendizagem.

Em virtude dessa influência, é necessário que esse conhecimento profissional esteja em constante desenvolvimento e, além disso, pesquisas a seu respeito.
Na literatura, alguns estudos têm apresentado modelos que buscam descrever aspectos desse conhecimento (BALL, THAMES; CARRILLO et al., 2013; FLORES-MEDRANO et al., 2014; PHELPS, 2008; SHULMAN, 1986), como o Conhecimento especializado do Professor de Matemática MTSK $^{1}$ (CARRILLO et al., 2013; FLORES-MEDRANO et al., 2014). Nesse modelo, todo conhecimento do professor de Matemática é especializado e essa especialização permite diferenciá-lo do conhecimento da pedagogia e psicologia geral, do conhecimento especializado do professor de outra disciplina e do conhecimento especializado de outro profissional da Matemática (FLORES-MEDRANO et al., 
2014).

Para fins analíticos, esse conhecimento é organizado em domínios e subdomínios que destacam suas características. Apesar do refinamento em subdomínios, Flores-Medrano et al. (2014) reforçam que o modelo não ignora o caráter sintético e integrado do conhecimento do professor e apresentam as crenças e as concepções dos professores a respeito do ensino e da aprendizagem da Matemática no centro do modelo, como um núcleo que permeia todo esse conhecimento.

Um dos domínios do MTSK é o conhecimento matemático $\left(\mathrm{MK}^{3}\right)$. Este domínio é composto por três subdomínios, sendo eses: conhecimento dos tópicos (KoT), que contempla o conhecimento aprofundado do conteúdo matemático; conhecimento da estrutura matemática (KSM), que diz respeito às conexões possíveis entre distintos conteúdos matemáticos; e conhecimento da prática matemática (KPM), que se refere a como explorar e gerar conhecimento em matemática (CARRILLO et al., 2013; FLORES-MEDRANO et al., 2014).

Já o outro domínio, conhecimento pedagógico do conteúdo (PCK), é composto pelos subdomínios: conhecimento do ensino de matemática (KMT), que diz respeito ao conhecimento de recursos, materiais e formas de apresentar o conteúdo potenciais para a aprendizagem; conhecimento das características da aprendizagem matemática (KFLM), como possíveis erros e obstáculos que os alunos podem apresentar em determinado conteúdo, bem como estratégias e vocabulário típicos utilizados por eles; e conhecimento dos padrões de aprendizagem matemática (KMLS), que contempla o conhecimento dos professores a respeito do que se deve ensinar e o que é esperado que o aluno alcance em cada nível de ensino (CARRILLO et al., 2013; FLORESMEDRANO et al., 2014).

Considerando os avanços do MTSK em relação a outros modelos de conhecimentos profissionais docentes, centra-se o tema deste artigo neste modelo, e se tem como objetivo apresentar um levantamento bibliográfico com o que tem sido investigado a respeito do MTSK em dissertações e teses brasileiras. Para isso, nas próximas seções se apresentam os aspectos metodológicos, os resultados e uma breve discussão seguida de algumas considerações a respeito do assunto.

\section{Material e Métodos}

Para a realização do levantamento bibliográfico se utiliza o Catálogo de Dissertações e Teses da Capes (Coordenadoria de
Aperfeiçoamento de Pessoal de Nível Superior $)^{4}$ e a Biblioteca Digital Brasileira de Teses e Dissertações (BDTD) ${ }^{5}$, com os disparadores "conhecimento especializado do professor de Matemática" e "MTSK" separadamente, tendo em vista que alguns autores podem optar por utilizar a sigla em inglês.

No Catálogo de Dissertações e Teses da Capes se encontram nove textos entre dissertações e teses, enquanto na Biblioteca Digital Brasileira de Teses e Dissertações são encontradas três que foram desconsiderados, pois já estavam entre os nove encontrados anteriormente.

Dos nove trabalhos, dois foram desconsiderados, pois se tratavam de uma tese de doutorado em Biologia celular e molecular e uma dissertação de mestrado em Medicina e Ciências da Saúde. Assim, o corpus $^{6}$ deste trabalho se estabeleceu em três dissertações de mestrado acadêmico e quatro teses de doutorado, apresentadas no quadro a seguir de acordo com o tipo de trabalho e a ordem de defesa.

Quadro 1 - Corpus de dissertações e teses

\begin{tabular}{|l|c|c|c|}
\hline \multicolumn{1}{|c|}{ Título } & $\begin{array}{c}\text { Tipo de } \\
\text { Trabalho }\end{array}$ & $\begin{array}{c}\text { Data de } \\
\text { defesa }\end{array}$ & Autor \\
\hline $\begin{array}{l}\text { Conhecimento } \\
\text { especializado } \\
\text { do professor de } \\
\text { matemática sobre } \\
\text { função no contexto } \\
\text { de uma experiência } \\
\text { prévia de lesson } \\
\text { study. }\end{array}$ & Dissertação & $13 / 07 / 2018$ & $\begin{array}{c}\text { Wellington } \\
\text { Rabello de } \\
\text { Araujo }\end{array}$ \\
\hline $\begin{array}{l}\text { Conhecimento } \\
\text { especializado de } \\
\text { professores de física: } \\
\text { uma proposta de } \\
\text { modelo teórico. }\end{array}$ & Dissertação & $29 / 08 / 2018$ & $\begin{array}{c}\text { Stela Silva } \\
\text { Lima }\end{array}$ \\
\hline $\begin{array}{l}\text { Conhecimento } \\
\text { especializado } \\
\text { de professores } \\
\text { de matemática } \\
\text { mobilizados em } \\
\text { um contexto de } \\
\text { planejamento de } \\
\text { ensino de divisões } \\
\text { de frações por meio } \\
\text { de resolução de } \\
\text { problemas. }\end{array}$ & Dissertação & $15 / 10 / 2018$ & $\begin{array}{c}\text { Yamamoto } \\
\text { Moral }\end{array}$ \\
\hline $\begin{array}{l}\text { Conhecimento } \\
\text { especializado para } \\
\text { ensinar divisão de } \\
\text { frações. }\end{array}$ & & & \\
\hline & & & $\begin{array}{c}\text { Tese } \\
\text { Junior }\end{array}$ \\
\hline
\end{tabular}

2 As siglas associadas ao modelo MTSK que serão apresentadas, a partir desse momento, se referem aos nomes dos subdomínios em inglês, quais sejam: Mathematical Knowledge (MK), Knowledge of Topics (KoT), Knowledge of the Structure of Mathematics (KSM), Knowledge of the Pratice of Mathematics (KPM), Pedagogical Content Knowledge (PCK), Knowledge of Features of Learning Mathematics (KFML), Knowledge of Mathematics Teaching (KMT) e Knowledge of Mathematics Learning Standards (KMLS).

$3 \mathrm{https} / / /$ catalogodeteses.capes.gov.br/catalogo-teses/\#!/. A busca foi realizada em 16/12/2019.

4 http://bdtd.ibict.br/vufind/. A busca foi realizada em 16/12/2019.

5 Conjunto de documentos e informações sobre determinado assunto. (MICHAELIS, 2020).

6 A data de defesa dessa tese foi retirada do site da biblioteca digital do Programa de Pós-Graduação em Educação em Ciências e Matemática, ao qual ela está associada, devido ao fato de não constar no arquivo digital original disponível no catálogo de dissertações e teses da Capes. 


\begin{tabular}{|c|c|c|c|}
\hline Título & $\begin{array}{l}\text { Tipo de } \\
\text { Trabalho }\end{array}$ & $\begin{array}{c}\text { Data de } \\
\text { defesa }\end{array}$ & Autor \\
\hline \begin{tabular}{|l|} 
Conhecimento \\
especializado do \\
professor dos anos \\
iniciais no âmbito da \\
multiplicação: uma \\
metassíntese de teses \\
produzidas entre 2001 \\
e 2012 em diferentes \\
contextos formativos.
\end{tabular} & Tese & $23 / 02 / 2018$ & $\begin{array}{c}\text { Rosana } \\
\text { Catarina } \\
\text { Rodrigues } \\
\text { de Lima }\end{array}$ \\
\hline \begin{tabular}{|l|} 
Conhecimento \\
especializado de \\
um formador de \\
professores de \\
matemática em início \\
de carreira: o ensino a \\
distância de derivada. \\
\end{tabular} & Tese & $23 / 10 / 2018$ & $\begin{array}{c}\text { Daiane } \\
\text { dos Santos } \\
\text { Correa } \\
\text { Cabanha }\end{array}$ \\
\hline \begin{tabular}{|l|} 
A construção \\
da identidade \\
profissional do \\
futuro professor de \\
matemática formado a \\
distância no contexto \\
do estágio.
\end{tabular} & Tese & $06 / 12 / 2018$ & $\begin{array}{c}\text { Priscila } \\
\text { Kabbaz } \\
\text { Alves da } \\
\text { Costa }\end{array}$ \\
\hline
\end{tabular}

Fonte: Dados da pesquisa.

Após a definição do corpus, foi realizada a leitura na íntegra de cada trabalho buscando identificar objetivos, contextos em que as investigações foram realizadas e resultados dessas pesquisas para se compreender como tem sido a utilização do MTSK em dissertações e teses e brasileiras. A partir destas leituras, tece-se, de maneira articulada na seção seguinte, uma síntese dos objetivos, contextos e resultados identificados, acompanhada de uma breve discussão sobre o tema.

\section{Resultados e Discussão}

Araújo (2018) buscou em sua dissertação identificar evidências de problematização de conhecimento especializado do professor de Matemática, sobre o tema de função no âmbito do Ensino Médio, em um contexto de experiência prévia de Lesson Study, que se deu no Grupo de Sábado da Universidade Estadual de Campinas (Unicamp). Nesse grupo, participam professores que ensinam Matemática e professores de Matemática dos mais variados anos de escolaridade, que se reúnem quinzenalmente aos sábados na Faculdade de Educação da Unicamp, sem obrigatoriedade ou vínculo institucional (ARAÚJO, 2018). Assim, o foco de análise do autor foram as

[...] experiências vivenciadas e os conhecimentos mobilizados por duas professoras que planejaram, implementaram e refletiram sobre uma tarefa inicial relativa à introdução ao estudo de função, tarefa que visava explorar analogicamente o conceito de função tendo como referência o funcionamento de uma Máquina. (ARAÚJO, 2018, p.55).

Para atingir seus objetivos foram utilizadas as gravações em áudio do momento de Análise/Reflexão realizado no Grupo de Sábado, em que as participantes relataram a experiência e discutiram a respeito da implementação da tarefa com os colegas do grupo. Nesse momento, segundo o autor, há uma socialização entre os participantes que enriquece o "movimento analítico e reflexivo dos professores com olhares mais amplos sobre as experiências" (ARAÚJO, 2018, p.59). Além disso, o autor utilizou narrativas produzidas pelas professoras participantes.

Como resultados, o trabalho de Araújo (2018) apresentou conhecimentos manifestados por essas professoras como, conceito de função como relações, e relação de dependência entre variáveis, que evidenciaram indícios de conhecimentos presentes nos subdomínios KoT e KSM, além do uso de termos, notações e representações que revelaram indícios de conhecimentos que compõem o KPM. Na dissertação, esses conhecimentos foram relacionados ao Pensamento Funcional, que não será aqui abordado por não se tratar do foco deste artigo.

Outros conhecimentos que também foram articulados com o Pensamento Funcional na pesquisa foram os presentes no domínio PCK. Quanto a esses, Araújo (2018) identificou manifestação de aspectos relacionados aos conhecimentos presentes em seus três subdomínios, como a utilização de livros didáticos como recursos (KMT), o conhecimento da sequenciação do tema e do currículo do Estado do São Paulo (KMLS), as dificuldades dos alunos em compreenderem os elementos de uma função, como o conjunto domínio, e as potencialidades da tarefa para auxiliar nessas dificuldades na introdução do conceito (KFLM).

Para o autor, o contexto da pesquisa possibilitou a conexão de um processo de desenvolvimento profissional com o MTSK, que auxilia a identificar dimensões do conhecimento do professor sobre o conteúdo matemático. $\mathrm{O}$ contexto ainda "influenciou e possibilitou a mobilização de conhecimentos especializados do professor de matemática ou sobre o conteúdo de função" (ARAÚJO, 2018, p.119). Contextos como esse, de grupos colaborativos com professores, em exercício da docência, podem ser propícios para a mobilização de conhecimentos profissionais docentes, que podem ser influenciados pela prática profissional e pela reflexão dessa prática. Consequentemente, são propícios para o desenvolvimento profissional, haja vista que tais conhecimentos podem ser considerados um aspecto desse desenvolvimento (CLIMENT et al., 2014; MARCELO, 2009; PASSOS et al., 2006;PONTE, 2014).

Tendo em vista um ambiente oportuno para o desenvolvimento profissional dos professores, Moral (2018) utilizou do contexto de uma oficina formativa para caracterizar o conhecimento especializado de professores de Matemática em um ambiente de planejamento de ensino, por meio da metodologia Resolução de Problemas em sua dissertação de mestrado. Ele destaca que a intenção era que nesse ambiente os participantes pudessem se manifestar sobre situações práticas (o ensino de divisão de frações através da Resolução de Problemas) e realizarem tarefas (planejamento de aula) com base em seus conhecimentos e experiências. 
Assim, observou e gravou a participação de quatro professores de Matemática dos Anos Finais do Ensino Fundamental em dois dias de realização de uma oficina, em que os participantes tinham como meta planejarem para ensinar divisão de frações por meio da Resolução de Problemas e, para isso, discutiam a respeito da metodologia, do conteúdo e do planejamento entre si e com o formador. A partir da análise da transcrição das gravações, como resultado o autor evidenciou mobilização de conhecimentos dos subdomínios do MTSK, o que lhe permitiu atingir seu objetivo.

De acordo com Moral (2018, p.65), durante todo o percurso apareceram características do KoT, subdomínio do KM, principalmente, nas discussões a respeito de "como fazer a divisão de frações", o que não aconteceu com os conhecimentos referentes aos subdomínios KSM e KPM que se mostraram ausentes nas análises do autor. Já em relação ao PCK, os conhecimentos manifestados com mais frequência se enquadram no subdomínio KFLM, quando os professores discutiam sobre as "fortalezas e dificuldades, formas de interação com o conteúdo matemático e interesses e expectativas" dos alunos em relação ao conteúdo.

Ainda, segundo o autor, o conhecimento referente ao subdomínio KMT também esteve presente, especialmente, pelas discussões a respeito da metodologia de ensino mais viável para o conteúdo, e o referente ao KMLS foi evidenciado quando professores se manifestaram sobre o nível de conhecimento do conteúdo esperado para determinada etapa escolar.

Uma das ações apontadas por Moral (2018) para serem realizadas após sua pesquisa consiste na proposição de ambientes de formação continuada, visando a utilização do MTSK, inclusive, como modelo de referência para o planejamento do trabalho a ser realizado nesses ambientes. A ideia do autor vai ao encontro do que escreve Flores-Medrano, Escudero-Ávila e Juarez (2018) a respeito da utilização do MTSK como quadro de referência para o conhecimento e, consequentemente, para o desenvolvimento profissional docente.

Falar sobre o desenvolvimento profissional do professor de matemática sem um quadro de referência que indique um estado ideal pode fazer com que as alterações relatadas sejam pouco usadas ou sistematizadas. Ter uma sólida estrutura de conhecimento profissional é um ponto de partida que nos permite ser uma referência, mas também ser um sistematizador nesse desenvolvimento profissional (FLORES-MEDRANO; ESCUDERO-ÁVILA; JUAREZ, 2018, p.377, tradução nossa).

Segundo Nascimento, Castro e Lima (2017, p. 490): "um dos grandes entraves ao desenvolvimento profissional de professores remete aos anos iniciais da carreira docente, apontados como os mais difíceis, face as lacunas oriundas da formação inicial.". Foi com foco em um formador de professores em início de carreira que Cabanha (2018) investigou o conhecimento especializado do formador em sua tese de doutoramento. Nessa, a autora objetivou caracterizar o conhecimento especializado de um formador de professores de Matemática, em início de carreira, especificamente, no ensino a distância de Derivada. Para isso, realizou uma entrevista inicial com o professor da disciplina de Cálculo I da Licenciatura em Matemática da Educação a Distância (EaD) da Universidade Federal de Mato Grosso do Sul (UFMS) e analisou o material publicado por ele na interação com os alunos no ambiente virtual de aprendizagem (AVA), em busca de indícios de conhecimento ou oportunidades em que pudesse explorar em uma próxima entrevista com o professor, realizada como etapa final da coleta de informações.

Como resultados, assim como Araújo (2018), Cabanha (2018) identificou características de todos os subdomínios do MTSK no conhecimento do professor formador em exercício da docência, detalhadas pela autora no trabalho. Ainda, apresentou algumas características da prática do professor, como oferecer mais atenção ao desenvolvimento do MK do que do PCK e a valorização de técnicas de resolução como uma possível influência de sua formação, e explanou sua satisfação com a pesquisa em poder refletir sobre conhecimento e, em especial, conhecimento de um formador de professores de Matemática a distância. A respeito da Educação a distância, Costa (2015, p.2) afirma que:

A EaD exige não só a mudança de postura do professor, mas também a do aluno, pois essa modalidade preza pela autonomia para aprender sempre, preparando o futuro professor para o exercício de sua profissão, de forma moderna, rica e dinâmica.

Assim, investigar conhecimentos profissionais docentes nessa modalidade de ensino se configura um amplo e promissor espaço de pesquisa, não só com o formador como também com os futuros professores. Com o olhar atento a $\mathrm{EaD}$, em sua tese de doutorado, Costa (2018, p.5) buscou compreender:

[...] as tensões vivenciadas na construção da identidade profissional do futuro professor de Matemática a partir da aprendizagem docente via participação em comunidades de prática e do conhecimento especializado do professor de matemática no contexto do estágio em um curso a distância.

Para isso, a autora elaborou dossiês de quatro licenciandos, dois do $8^{\circ}$ semestre e dois do $6^{\circ}$ semestre do curso de Licenciatura em Matemática da Universidade Estadual de Ponta Grossa, oferecido a distância em parceria com a Universidade Aberta do Brasil. Esses dossiês foram compostos de questionários, entrevistas e materiais disponibilizados no AVA, como tarefas, fóruns e relatórios, e serviram de base para que a autora construísse narrativas individuais de cada sujeito, utilizando das lentes teóricas adotadas para analisálas, entre as quais estava o MTSK.

Em relação ao $\mathrm{MTSK}^{7}$, Costa (2018, p.189) afirma

7 Outros resultados são apontados no trabalho relacionados à identidade profissional, as comunidades de prática, ao AVA e a estrutura do curso, não apresentados aqui por não serem foco desse trabalho. Caso o leitor tenha curiosidade, recomenda-se acesso ao texto da autora na íntegra. 
que este auxilia a compreender "como o futuro professor constrói seu conhecimento especializado para que ajude-o a planejar, prever, aprender a improvisar" no seu futuro campo de atuação profissional, e afirma que o modelo pode ajudar a pensar o curso que foi contexto de sua pesquisa de modo que MK e PCK sejam integrados, o que, segundo ela, ainda não acontecia. Também diz ser evidente no discurso dos sujeitos a alta preocupação com os conhecimentos do subdomínio KoT e a incidência reduzida dos conhecimentos referentes aos subdomínios KSM e KPM, semelhante ao que evidenciou Moral (2018). Quanto ao PCK, o estudo revela maior frequência de identificação dos conhecimentos dos subdomínios KMT e KFLM, e conclui que, de maneira geral, a "formação como está organizada não reflete a formação como um todo dentro do MTSK". (COSTA, 2018. p.191).

Além disso, a autora escreve sobre a possibilidade de propor e executar uma disciplina de estágio na perspectiva do MTSK, desejo compartilhado com os próprios autores do modelo, pois segundo Montes, Contreras e Carrillo (2013), em suas pesquisas, pretendem destacar seu potencial do MTSK ao desenvolverem propostas de formação nos contextos de formação inicial e continuada de professores de Matemática de qualquer nível educacional.

Atento não somente à formação inicial, como também a continuada, e com a intenção de caracterizar o conhecimento mobilizado não só pelo futuro professor, mas também pelos professores em exercício da docência, Moriel Junior (2014) investigou futuros professores e professores em exercício da docência em sua tese.

Nessa, o autor buscou caracterizar o conhecimento especializado para ensinar divisão de frações mobilizado por professores e licenciandos em Matemática em um contexto de formação. Para isso, gravou duas oficinas a respeito de como ensinar divisão de frações, realizadas com 54 participantes no âmbito do projeto Observatório da Educação ${ }^{8}$ da Universidade Federal do Mato Grosso em Cuiabá, em que observou dois licenciandos e duas professoras de Matemática para obter indícios de conhecimento especializado, confirmados ou não, posteriormente, pelo autor, a partir de entrevistas semiestruturadas.

$\mathrm{O}$ autor relata que nas oficinas foi priorizado um ambiente de colaboração entre os participantes, em que pudessem participar e elaborar respostas com base nas experiências pessoais e no próprio conhecimento, socializando-as com todo o grupo para que pudessem discutir possibilidades de respostas construídas a partir da literatura.
A partir das informações coletadas, Moriel Junior (2014) configurou o MTSK da divisão de frações, elencando características de cada categoria do conhecimento especializado para ensinar divisão de frações, contemplando todos os subdomínios do modelo. Segundo ele, o MTSK da divisão de frações possibilita que professores e futuros professores ampliem a compreensão sobre o próprio conhecimento, e que formadores de professores desenvolvam atividades que oportunizem a construção desse conhecimento especializado.

Esse conhecimento do professor, defendido como especializado, não possui essa característica apenas na Matemática. Lima S. (2018) busca, em sua dissertação, propor um modelo de conhecimento especializado de professores de Física, PTSK $^{9}$, que tenha como referencial teórico a base conceitual do modelo MTSK. Para propô-lo, a autora inicialmente realizou uma transposição direta do MTSK, descrevendo os domínios e subdomínios do que chamou de PTSK Transposto, além das categorias do subdomínio KoT desse modelo. Após isso, selecionou artigos, dissertações e teses que continham PaP-eRs ${ }^{10}$, sigla que se refere ao "Relatório da Experiência Profissional Pedagógica", descrito por ela como documento que emerge da prática real dos professores, no qual um episódio de ensino é reconstruído em forma comunicável contendo descrições detalhadas sobre situações na prática de ensino de conteúdo específico. (LIMA, 2018), e analisou nesses trabalhos a mobilização de conhecimentos com base no PTSK Transposto.

Feita a análise, utilizou dos seus resultados para validar o modelo que havia descrito e propor, de fato, a descrição dos domínios e subdomínios do modelo de conhecimento PTSK, além da descrição das categorias do subdomínio conhecimento dos tópicos de Física. Assim, Lima S. (2018) caracteriza o PTSK descrevendo o que englobaria cada um dos domínios, conhecimento de Física e conhecimento pedagógico do conteúdo, bem como todos os seis subdomínios, nomeadamente: conhecimento dos tópicos de Física; conhecimento da estrutura da Física; conhecimento da prática da Física; conhecimento das características da aprendizagem de Física; conhecimento do ensino de Física e conhecimento dos parâmetros da aprendizagem de Física; além das categorias do subdomínio conhecimento dos tópicos da Física, a saber: leis, fenômenos e conceitos; linguagem Matemática; registros de representações; modelos; experimentação; e aplicação.

A autora ressalta a necessidade de pesquisas futuras detalharem as descrições das categorias dos outros

8 O projeto fez parte do Programa Observatório da Educação financiado pela Coordenadora de Aperfeiçoamento de Pessoal de Nível Superior, o Instituto Nacional de Estudos e Pesquisas Educacionais Anísio Teixeira e a Secretaria de Educação Continuada, Alfabetização, Diversidade e Inclusão, e era uma parceria entre Universidade Estadual Paulista (UNESP, campus Bauru - SP), Universidade Estadual de Mato Grosso - (UNEMAT, campus Cáceres - MT) e Universidade Federal de Mato Grosso (UFMT, campus Cuiabá - MT), cujo trabalho está associado (MORIEL JUNIOR, 2014). Ele contava com a participação de alunos das escolas atendidas, professores da Educação Básica, licenciandos de Matemática e de Pedagogia, mestrandos, doutorandos e um professor doutor que o coordenava.

9 De acordo com Lima (2018), Physics Teacher's Specialized Knowledge.

10 Professional and Pedagogical experience Repertoire. 
subdomínios do modelo e a de validação das conclusões obtidas em cenários práticos de interação com os professores.

Em sua tese de doutorado, Lima R. (2018) buscou investigar a abordagem dada por pesquisas acadêmicas brasileiras ao conhecimento especializado do professor que ensina matemática (PEM), nos anos iniciais de escolarização, em relação à multiplicação e, para isso, realizou um mapeamento relativo ao PEM nos anos iniciais do Ensino Fundamental partindo de um universo de 858 trabalhos até definir o corpus de três teses de doutorado, utilizadas para a realização de um estudo de metassíntese.

A autora relata que sua investigação evidenciou diferentes aspectos do conhecimento especializado do professor que ensina Matemática nos anos iniciais a respeito de multiplicação, tanto no âmbito da formação inicial, quanto da continuada. Seus resultados revelam também a complexidade da relação entre o domínio desse conhecimento pelos formadores de professores e as estratégias e práticas utilizadas com futuros professores na formação inicial e com professores na formação continuada para que possam se apropriar desse conhecimento. Ainda, Lima R. (2018, p.161) ressalta, a partir da identificação de "perspectivas diferenciadas e complementares para o tratamento do tema da multiplicação", que esse conhecimento especializado não pode ser prescritivo, mas deve ganhar visibilidade e compreensão a partir da prática do professor em exercício, no caso da formação continuada, ou na exploração de processos e conceitos passados adquiridos por futuros professores e trazidos para o contexto da formação inicial.

Em síntese, destaca-se que as dissertações e teses utilizaram o modelo, principalmente, para identificar e caracterizar o conhecimento especializado de professores em relação a um determinado conteúdo, especificamente, divisão de frações (MORAL, 2018; MORIEL JUNIOR, 2014), função (ARAÚJO, 2018) e derivadas (CABANHA, 2018), e para compreender a construção desse conhecimento por futuros professores no contexto do estágio de um curso a distância (COSTA, 2018). Nos trabalhos de caráter teórico, o MTSK foi utilizado como suporte para a construção de um modelo de conhecimento para a Física (LIMA S., 2018) e como ferramenta de análise em um estudo de metassíntese a respeito da abordagem dada ao conhecimento especializado do professor, nos anos iniciais, em relação à multiplicação (LIMA R., 2018).

\section{Conclusão}

Busca-se com este artigo investigar a utilização do MTSK em dissertações e teses brasileiras. Para isso, realizou-se um levantamento bibliográfico em dois bancos de dados e a leitura dos sete trabalhos que compuseram o corpus. Com base nestas leituras, para atingir o objetivo, procura-se evidenciar, de maneira sintética, o objetivo, o contexto e o resultados de cada trabalho.

Destaca-se a abordagem predominantemente analítica adotada nas dissertações e teses em relação ao MTSK, por meio da qual se constata a utilização do modelo para identificar ou caracterizar conhecimentos relativos a um conteúdo específico, para compreender a construção do conhecimento especializado por futuros professores no contexto do estágio de um curso a distância, e para investigar e analisar a abordagem dada ao conhecimento especializado do professor em outras pesquisas. Além disso, o MTSK proporcionou base teórica para a elaboração de um modelo de conhecimento semelhante para a Física.

Os resultados de algumas pesquisas acenaram para a possibilidade de utilização do modelo para regular propostas de estágio e processos de formação continuada. Já o emprego do modelo na pesquisa que envolvia o ensino a distância ajudou a problematizar também aspectos dessa modalidade de ensino.

As pesquisas de campo foram realizadas com professores e futuros professores em ambientes virtuais do ensino a distância, e em contextos presenciais identificados como oficinas formativas e grupos colaborativos, que influenciaram na oportunidade de mobilização dos conhecimentos. Dos trabalhos realizados, em contextos presenciais, apenas um teve a participação de futuros professores e os demais foram realizados, exclusivamente, com professores em formação continuada, o que evidenciou a ausência e a necessidade de investigações a respeito do MTSK na modalidade presencial da formação inicial.

Dadas as potencialidades do modelo para identificar e oportunizar a construção de conhecimentos especializados por professores e futuros professores, que ensinam Matemática, considera-se importante a sua utilização para orientar processos formativos nos mais variados contextos de formação profissional docente.

Um fato que chama atenção, considerando o levantamento bibliográfico, consiste na mobilização mais frequente do subdomínio KoT e menos frequente dos subdomínios KSM e KPM do domínio MK nos resultados de alguns dos trabalhos, o que acena para a possibilidade de pesquisas futuras investigarem possíveis causas desse fato.

Assim como alguns dos trabalhos analisados, se espera que as pesquisas em Educação Matemática cada vez mais discutam as necessidades dos professores e ressaltem a importância de valorização da profissionalização docente, do respeito à profissão, e de políticas públicas reiterem o conhecimento do professor como especializado.

\section{Referências}

ARAÚJO, W.R. Conhecimento especializado do professor de Matemática sobre função no contexto de uma experiência prévia de lesson study. 2018. Dissertação (Mestrado em Educação ) Universidade Estadual de Campinas, Campinas, 2018.

BALL, D.L.; THAMES, M.H.; PHELPS, G. Content Knowledge for teaching: what makes it special? J. Teacher Educ., v.59, n.5, p.389-407, 2008. doi: 10.1177/0022487108324554.

BRASIL. Ministério da Educação. Secretaria de Educação Básica. Orientações Curriculares para o Ensino Médio: Ciências 
da Natureza, Matemática e suas Tecnologias. Brasília: MEC/ SEB, 2006.

BRASIL. Ministério da Educação, Secretaria de Educação Média e Tecnológica (Semtec). PCN + Ensino médio: orientações educacionais complementares aos Parâmetros Curriculares Nacionais - Ciências da Natureza, Matemática e suas Tecnologias. Brasília: MEC/Semtec, 2002.

CABANHA, D.S.C. Conhecimento Especializado de um Formador de Professores de Matemática em início de carreira: o ensino a distância de Derivada. 2018. Tese (Doutorado em Geociências e Ciências Exatas) - Universidade Estadual Paulista, Rio Claro, 2018.

CARRILLO, J. et al. Determining specialised knowledge for mathematics teaching. In: UBUZ, B. et al. (Ed.). VIII CONGRESS OF THE EUROPEAN SOCIETY FOR RESEARCH IN MATHEMATICS EDUCATION (CERME 8). 8. Antalya, Turkey: Middle East Technical University, Ankara, p.2985-2994. 2013.

CLIMENT, N. et al. El conocimiento del profesor para la enseñanza de la Matemática. In: AGUILAR, Á. et al. Un marco teórico para el conocimiento especializado del profesor de matemáticas. Huelva: Universidad de Huelva Publicaciones, p. 43-70, 2014.

CORPUS. In: MICHAELIS dicionário brasileiro da Língua Portuguesa. São Paulo: Editora Melhoramentos, 2020. Disponível em: $\quad<$ https://michaelis.uol.com.br/moderno-portugues/busca/ portugues-brasileiro/corpus/> Acesso em: 01 mar. 2020.

COSTA, P. K. A. O desenvolvimento profissional e a formação inicial do professor em um curso de licenciatura em matemática a distância: uma análise narrativa na perspectiva dos egressos. In: ENCONTRO BRASILEIRO DE ESTUDANTES DE PÓSGRADUAÇÃO EM EDUCAÇÃO MATEMÁTICA, 2015, Juiz de Fora. Anais [...]. Juiz de Fora, 2015. Disponível em: <http:// www.ufjf.br/ebrapem2015/files/2015/10/gd7_priscila_kabbaz. pdf $>$ Acesso em: 11 mai. 2020.

COSTA, P.K.A. Tensões e contribuições do estágio curricular na constituição da identidade profissional do licenciando em Matemática na EaD. Campinas: Universidade Estadual de Campinas, 2018.

FLORES-MEDRANO, E. et al. Nuestra modelación del conocimiento especializado del profesor de matemáticas, el MTSK. In: AGUILAR, Á. et al. Un marco teórico para el conocimiento especializado del profesor de matemáticas. Huelva: Universidad de Huelva Publicaciones, p. 57-72, 2014.
FLORES-MEDRANO, E.; ESCUDERO-ÁVILA, D.; JUÁREZ, J. A. Desarollo del conocimiento de la práctica matemática em docentes de Matemáticas. In: CONGRESO IBEROAMERICANO DE EDUCACIÓN MATEMÁTICA. CIBEM. Libro de actas, Madrid, p. 376-383, 2018.

LIMA, R.C.R. Conhecimento especializado do professor dos anos iniciais no âmbito da multiplicação: uma metassíntese de teses produzidas entre 2001 e 2012 em diferentes contextos formativos. 2018. Tese (Doutorado em Educação) - Universidade Estadual de Campinas, Campinas, 2018.

LIMA, S.S. Conhecimento especializado de professores de física: uma proposta de modelo teórico. 2018. Dissertação (Mestrado em Ensino) - Instituto Federal de Educação, Ciência e Tecnologia de Mato Grosso, Cuiabá, 2018.

MARCELO, C. Desenvolvimento Profissional Docente: passado e futuro. Sísifo. Rev. Ciênc. Educ., v. 8, p.7-22, 2009.

MONTES, M.A.; CONTRERAS, L.C.; CARRILLO, J. Conocimiento del profesor de matemáticas: Enfoques del MKT y del MTSK. In: BERCIANO, G. et al. Investigación en Educación Matemática XVII, Bilbao, p. 403-410, 2013.

MORAL, G.C.Y. Conhecimento especializado de professores de matemática mobilizados em um contexto de planejamento de ensino de divisões de frações por meio de resolução de Problemas. 2018. Dissertação (Mestrado em Ensino) - Universidade de Cuiabá, Cuiabá, 2018.

MORIEL JUNIOR, J. G. Conhecimento especializado para ensinar divisão de frações. 2014. Tese (Doutorado em Educação em Ciências e Matemática) - Universidade Federal de Mato Grosso, Cuiabá, 2014.

NASCIMENTO, F.J.; CASTRO, E.R.; LIMA, I.P. Desenvolvimento profissional de professores de matemática iniciantes: contribuição do PIBID. Rev. Eletr. Educ., v.11, n.2, p.487-504, 2017. doi: http://dx.doi.org/10.14244/198271991962

PASSOS, C.L.B. et al. Desenvolvimento profissional do professor que ensina Matemática: Uma meta-análise de estudos brasileiros. Quadrante, v.15, n.1/2, p.193-219, 2006.

PONTE, J.P. Formação do professor de Matemática: perspectivas atuais. In: PONTE, J.P. Práticas profissionais dos professores de matemática. Lisboa: Instituto de Educação da Universidade de Lisboa, 2014. p. 343-360.

SHULMAN, L.S. Those who understand: knowledge growth in teaching. Educ. Res., v.15, n.2, p.4-14, 1986. 